\title{
A MICROMACHINED WIRELESS GAMMA RADIATION DETECTOR USING BULK METAL CONVERSION LAYERS
}

\author{
Christine K. Eun and Yogesh B. Gianchandani
}

Department of Electrical Engineering and Computer Science, University of Michigan, Ann Arbor

\begin{abstract}
This paper reports a micromachined wireless gamma radiation sensor. The gas-based detector is comprised of a patterned bulk metal conversion layer assembled on top of a pair of thin-film copper electrodes electroplated on a glass substrate. The metal layer converts gamma radiation into photoelectrons, which ionize the sensing gas. A bias between the electrodes creates an electric field that accelerates the charged particles, resulting in a current pulse. These current pulses generate a wideband signal extending into the $\mathrm{GHz}$ frequency range. Experiments were conducted in two gas environments: in an $\mathrm{N}_{2} / \mathrm{Ne}$ mixture at 640 Torr and in air at 760 Torr. Two types of metals (platinum-rhodium and tungsten) for the conversion layer were evaluated using $1.0 \mu \mathrm{Ci}$ gamma sources of ${ }^{65} \mathrm{Zn}$ and ${ }^{22} \mathrm{Na}$. By using a trench pattern $(1.5 \mathrm{~mm} \times 1.8 \mathrm{~mm})$, machined in a $200 \mu \mathrm{m}$-thick Pt-Rh metal layer, the count rate from the detector exceeded $28 \mathrm{kcpm}$ and showed $>10 \mathrm{X}$ improvement in sensing when compared to an un-patterned metal layer. The detector operated under partial pressure in the nitrogen/neon mixture. Other metal conversion patterns and stack configurations were also investigated.
\end{abstract}

\section{INTRODUCTION}

Sensing gamma radiation is important for many homeland security applications. Other forms of radiation (alphas and betas) have limited ranges and can be shielded more easily. For example, sheets of paper easily block alpha particles, while beta particles have ranges on the order of meters in air. Gamma-rays are highenergy photons that are emitted from many dangerous radioisotopes. Depending on their initial energy, they can travel up to tens of meters in air and require thick layers of lead for shielding. However, they are difficult to detect because of their low probability of interaction with matter. Miniaturized wireless gamma sensors could someday be used in a network configuration to monitor areas with high pedestrian traffic (e.g. shopping malls, football stadiums, subway stations). They would offer ultraportability and low power consumption, enabling rapid deployment and reconfiguration.

A miniaturized gas-based beta sensor with wireless capability has been reported in [1]. It is comprised of thin-film metal electrodes deposited on a glass substrate surrounded by a sensing gas mixture. Beta particles (or high-energy electrons) ionize the gas atoms and initiate electron avalanche breakdown, which results in a current pulse. These current pulses have been shown to generate a wireless signal for use in networking applications.

Gamma radiation is not detected directly by gas-based devices because the low density of gas provides low probability of interaction with the incident radiation. Conventional Geiger counters detect gamma radiation by the photoelectric effect. Gamma photons bombard the metal encapsulation of the sensing gas, producing photoelectrons within the Geiger tube, that avalanche and create current pulses. The use of metals to convert gamma-rays into photoelectrons is well established [2-6].

This paper presents a micromachined gas-based wireless gamma radiation detector that uses a bulk metal layer for gammato-photoelectron conversion. In particular, it explores the impact of thickness and structural shape of the metal conversion layer on two device configurations.

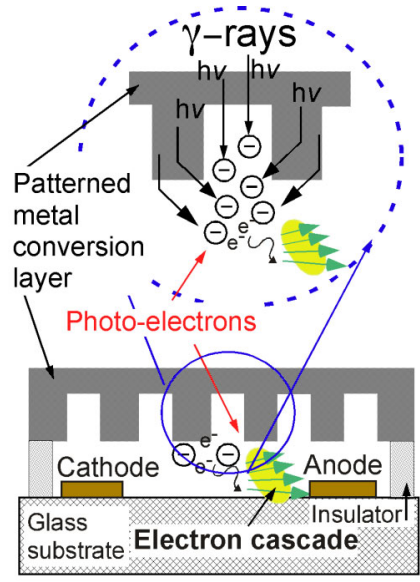

Design A

(a)

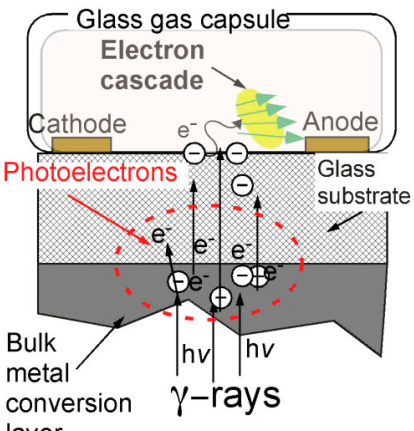

layer (b)

Fig. 1: (a) Design A - Cross-section of gamma detector with patterned bulk metal conversion layer. As gamma radiation interacts with the metal conversion layer, the generated photoelectrons ionize the surrounding gas between the electrodes, initiating a gas discharge and current pulse. Design A can facilitate a higher collection efficiency of these scattered events. (b) Cross-section of preliminary device structure (Design B) with an un-patterned metal layer. The photoelectrons penetrate through the glass substrate to ionize the gas atoms.

\section{DEVICE CONCEPTS AND OPERATION}

One device structure (Design A) consists of a glass substrate with electroplated copper electrodes and assembled on top of it, a micromachined conversion layer patterned from bulk metal (Fig. 1a). When gamma radiation interacts with the metal conversion layer (MCL), photoelectrons are emitted and ionize the surrounding gas. An electric field between the electrodes accelerates the electrons, leading to an avalanche breakdown measured as a current pulse. Two machining patterns that increase the exposed surface area for the Design A MCL are proposed: (1) A groove or trenchstyle pattern (TRENCH MCL) and (2) a square well or columnstyle pattern (COLUMN MCL). These structures can enhance the generation and collection efficiency of photoelectrons. Increasing the metal surface area exposed to the sensing gas can decrease photoelectron re-absorption probability in the MCL [2, 3].

Design $\boldsymbol{B}$ (Fig. 1b) is a simpler configuration that uses an unpatterned MCL (SOLID MCL) to achieve the basic conversion efficiency without structural enhancement. (In this, the MCL is located on the backside of the glass substrate.) The photoelectrons travel through the back of the glass substrate and ionize the gas atoms located near the electrodes.

Another choice for the substrate material is mica. In conventional Geiger counters, alpha particles and lower energy beta particles must travel through a thin mica window $(70-100 \mu \mathrm{m})$ in order to reach the sensing gas. Factors that need to be considered in choosing an appropriate substrate material include stiffness, yield strength, temperature coefficient, and charging issues. 
The emission and scattering processes are statistically determined by gamma energy (Fig. 2). Gamma radiation ionizes via three main processes: (1) the photoelectric effect, in which the gamma-ray imparts all its energy to a photoelectron, which scatters at an angle determined by the gamma energy (low-energy photons); (2) Compton scattering, in which the gamma-ray imparts only a portion of its energy to the photoelectron resulting in multiple photoelectrons per gamma-ray (medium-energy photons); and (3) pair production, in which the energy of the gamma radiation is converted to an electron/positron pair (high-energy photons).

Higher-density metals (e.g. tungsten and platinum-rhodium) are desirable for the MCL because of their higher interaction probabilities. Figure 3 shows the attenuation coefficient as a function of photon energy for $\mathrm{W}$ and Pt-Rh [7]. Having similar densities and excitation energies, they share similar collision processes. At higher photon energies ( $>1 \mathrm{MeV})$, Compton scattering dominates and results in multiple photoelectrons generated in the metal layer per gamma-ray. Figure 4 compares the theoretical range of photoelectrons through various media such as air, Pyrex glass, and metals [8]. Photoelectrons have similar theoretical ranges in $\mathrm{W}$ and $\mathrm{Pt}-\mathrm{Rh}$. Even though photon interactions increase with metal thickness, the resulting photoelectrons have a higher probability of re-absorption back into the metal layer, instead of being ejected out. The optimal relationship is to have photoelectron mean-free-range comparable to the MCL thickness.

Table I lists gamma-emitting radioisotopes used in the testing phase of this effort: $1.0 \mu \mathrm{Ci}{ }^{65} \mathrm{Zn}$ and $1.0 \mu \mathrm{Ci}{ }^{22} \mathrm{Na}$. Both are relatively high-energy gamma radiation sources that emit beta particles in conjunction with gamma radiation. Unlike beta radiation that is emitted over a spectrum of radiation energy levels, gamma radiation is emitted at specific energies. The interactions of $\mathrm{W}$ and $\mathrm{Pt}-\mathrm{Rh}$ with these sources are primarily the photoelectric effect, and Compton scattering.

\begin{tabular}{|c|c|}
\hline $\begin{array}{l}\text { 1. Photoelectric effect (low } \mathrm{E}) \\
\text { Gamma-ray }\end{array}$ & \\
\hline $\begin{array}{l}\text { 2. Compton scattering (medium } E) \\
\text { Gamma-ray } \\
\left.\text { Gve } E_{2}\right)\end{array}$ & $\begin{array}{l}\text { Fig. 2: Collision } \\
\text { processes for gamma } \\
\text { radiation interacting in } \\
\text { a medium. The photon } \\
\text { energy determines the }\end{array}$ \\
\hline $\begin{array}{l}\text { 3. Pair production (high } \mathrm{E}) \\
\left(\mathrm{E}_{1}>1.022 \mathrm{MeV}\right) \\
\text { Gamma-ray Electron }\left(\frac{\mathrm{E}_{1}-1.022 \mathrm{MeV}}{2}\right) \\
\text { Free electron }\end{array}$ & $\begin{array}{l}\text { type of collision as well } \\
\text { as the scattering angle } \\
\text { of the resulting } \\
\text { photoelectron. }\end{array}$ \\
\hline $\mathrm{h} v(\mathrm{E}=0.51 \mathrm{MeV})$ & \\
\hline
\end{tabular}

Table I: List of gamma-emitting radioisotopes by type and energy of emissions. $\beta$-particles are commonly released in conjunction with gamma radiation.

\begin{tabular}{|c|c|c|c|c|}
\hline *Most frequent emission & \multicolumn{2}{|c|}{$\begin{array}{c}\text { Gamma Energies } \\
(\mathrm{MeV})\end{array}$} & \multicolumn{2}{|c|}{$\begin{array}{c}\text { Beta Energies } \\
(\mathrm{MeV})\end{array}$} \\
\hline Isotope & $\mathrm{E}_{\mathrm{MIN}}$ & $\mathrm{E}_{\mathrm{MAX}}$ & $\mathrm{E}_{\mathrm{MIN}}$ & $\mathrm{E}_{\mathrm{MAX}}$ \\
\hline $\mathrm{Na}-22$ & \multicolumn{2}{|c|}{$* 1.274$} & \multicolumn{2}{|c|}{$* 0.546$} \\
\hline $\mathrm{Zn}-65$ & 0.345 & $* 1.115$ & & 30 \\
\hline
\end{tabular}

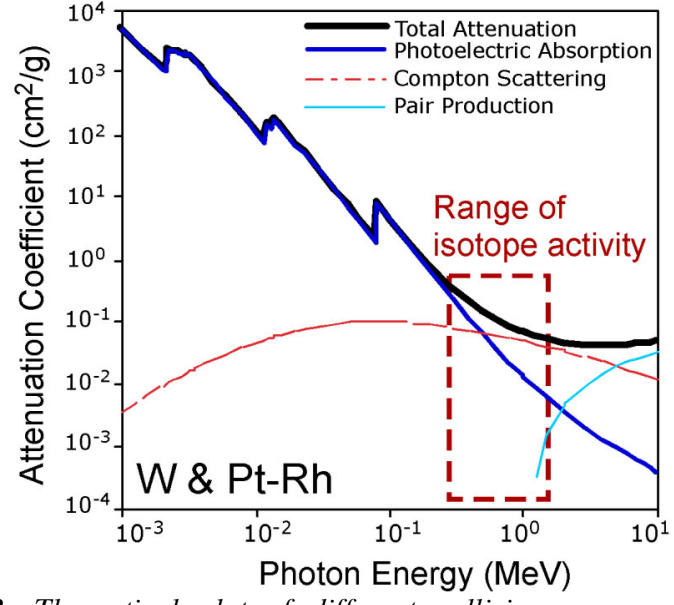

Fig. 3: Theoretical plot of different collision processes as a function of gamma energy for tungsten and platinum-rhodium [7]. Atten. coefficient has been normalized by material density $\left(\mathrm{g} / \mathrm{cm}^{3}\right)$.

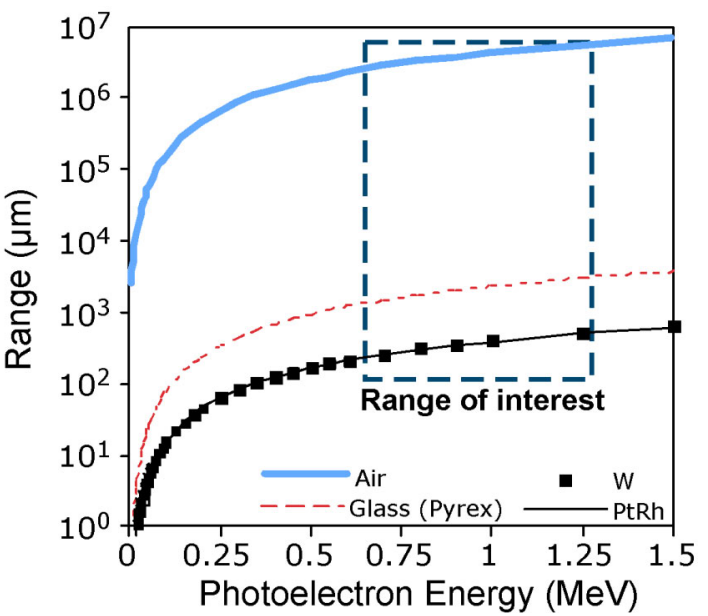

Fig. 4: Theoretical range of electrons through tungsten (W), platinum-rhodium (Pt-Rh), glass and air [8]. The thickness of the metal layer should be less than the range of the photoelectrons in order to maximize interaction with the gas.

\section{FABRICATION}

The Pt-Rh metal conversion layer is patterned by batch mode micro-electro-discharge machining $(\mu \mathrm{EDM})$. Figure 5 shows the machining process. In $\mu \mathrm{EDM}$, controlled spark discharges $(80 \mathrm{~V}$, $100 \mathrm{pF}, 5 \mathrm{~kW}$ ) between a tool cathode and workpiece anode thermally erode conductive material. In batch mode, the entire pattern is machined in parallel, increasing throughput. The 175 $\mu$ m-tall copper tool is fabricated with a PMMA LIGA process but an SU-8 process can also be used. A $200 \mathrm{~nm}$ silicon coating is deposited on the tool and selectively removed at the top to limit the effects from debris buildup [9].

The finished COLUMN MCL pattern is an $8 \times 8$ array of 110 $\mu \mathrm{m}$ square holes with $90 \mu \mathrm{m}$ spacing, giving a total active area of $1.5 \mathrm{~mm} \times 1.5 \mathrm{~mm}$. To form the TRENCH MCL, the same tool was dithered laterally by $150 \mu \mathrm{m}$ during machining, giving an active area of $1.5 \mathrm{~mm} \times 1.8 \mathrm{~mm}$. Each pattern was machined to a depth of $110 \mu \mathrm{m}$ in a $200 \mu \mathrm{m}$-thick Pt-Rh foil. Assembly of the final device includes attaching an insulator layer (63 $\mu \mathrm{m}$-thick Kapton foil) followed by alignment of the patterned conversion metal to the electrode structure. Figure 6 shows photographs of the assembled detectors with the (a) TRENCH MCL and (b) COLUMN MCL. 
Expose \& Develop PMMA

Electroplate \& lap copper

Strip PMMA

Sputter Silicon

Reverse $\mu \mathrm{EDM}$ top of $\mathrm{Si}$, then Forward $\mu \mathrm{EDM}$ $\mathrm{Pt} / \mathrm{Rh}$

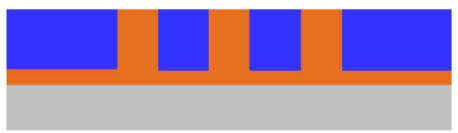

Sandwich insulator with glass substrate and patterned electrodes

Align Pt/Rh with electrodes

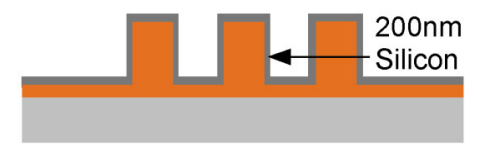
$\mu E D M$ Discharges
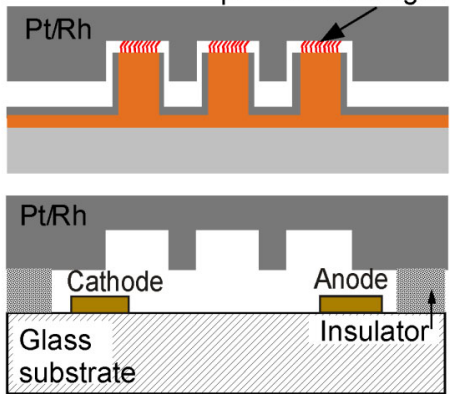

Fig. 5: Process flow for batch-mode micro-electro-discharge machining to pattern the TRENCH MCL and COLUMN MCL for Design A.

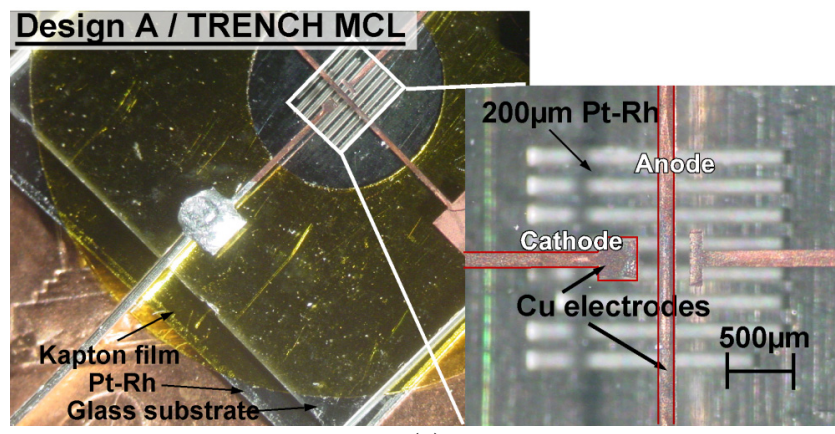

(a)

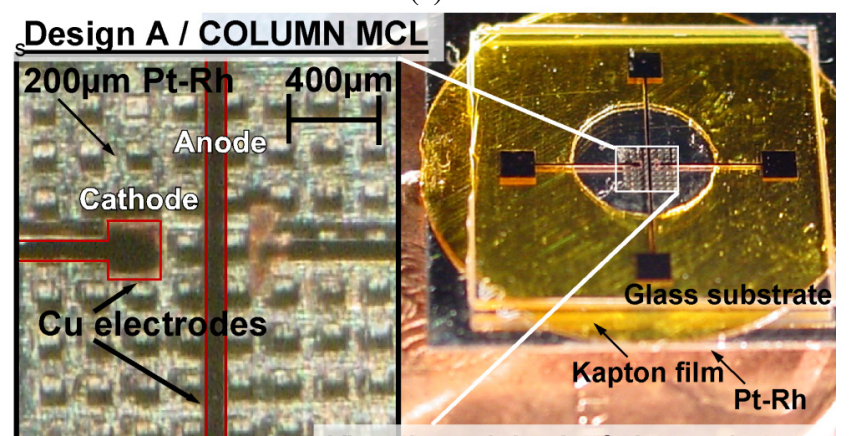

View through back of glass substrate

(b)

Fig. 6: Photograph of assembled Design A with $200 \mu m$-thick PtRh layer using the (a) TRENCH MCL and (b) COLUMN MCL. Shown through the back of the glass substrate.

\section{RESULTS AND DISCUSSION}

To determine the effectiveness of the MCL, the gamma sensors were tested with and without the metal layer present. Since each source emits both beta particles and gamma radiation, without the MCL for gamma conversion, the count rate should be primarily due to beta particles alone. With the MCL present, the count rate should be higher because the photoelectrons (from the gamma converter) can be sensed in addition to the beta particles.
Experiments were conducted for Design A and Design $B$ in two sensing gases and pressures: air at 760 Torr and a nitrogen-to-neon gas ratio of 5:2 at 640 Torr. Also, two bulk metals (50 $\mu$ m-thick W and $200 \mu \mathrm{m}$-thick Pt-Rh) and two radioisotopes $\left(1.0 \mu \mathrm{Ci}{ }^{22} \mathrm{Na}\right.$ and $1.0 \mu \mathrm{Ci}{ }^{65} \mathrm{Zn}$ ) were evaluated. $\mathrm{W}$ and $\mathrm{Pt}-\mathrm{Rh}$ have similar bulk properties and consequently have comparable conversion efficiencies. Finally, the performance of two machined patterns (TRENCH MCL, COLUMN MCL) in Pt-Rh were evaluated and compared to the performance of a SOLID MCL of Pt-Rh.

Design A with the Pt-Rh TRENCH MCL was evaluated in the presence of ${ }^{22} \mathrm{Na}$, in air at 760 Torr and in the $\mathrm{N}_{2} / \mathrm{Ne}$ sensing gas mixture under partial pressure. The count rate with the $\mathrm{N}_{2} / \mathrm{Ne}$ gas mixture ( $>28$ kcounts-per-minute) appeared to be almost twice the count rate in air. The operating voltage was fixed at $900 \mathrm{~V}$. Figure 7 compares the relative count ratios for Design A, while detecting ${ }^{22} \mathrm{Na}$. Relative count ratio is defined as the count rate with a patterned MCL over the count rate with the SOLID MCL. The TRENCH MCL and COLUMN MCL in $200 \mu \mathrm{m}$-thick Pt-Rh were evaluated at two different pressures (640 Torr, 760 Torr) and sensing gases $\left(\mathrm{N}_{2} / \mathrm{Ne}\right.$ mixture, air). The TRENCH MCL demonstrated a $>10 \mathrm{X}$ improvement in count rate over the SOLID MCL, while the COLUMN MCL showed $\approx 6 \mathrm{X}$ increase. The TRENCH MCL showed comparable performance in count ratios $(>10 X)$ in the $\mathrm{N}_{2} / \mathrm{Ne}$ mixture at 640 Torr and in air at 760 Torr.

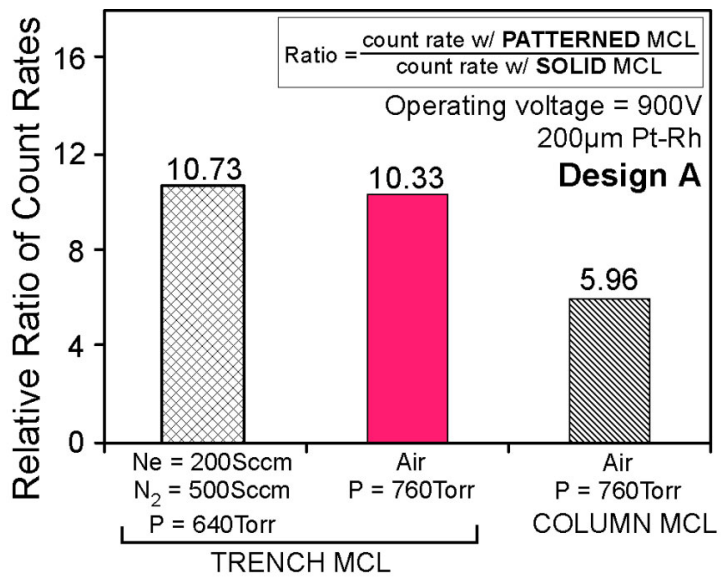

Fig. 7: Comparison of count ratios for Design A with two different machined patterns (TRENCH MCL, COLUMN MCL) in Pt-Rh and evaluated at two different pressures (640 Torr, 760 Torr) and sensing gases $\left(\mathrm{N}_{2} / \mathrm{Ne}\right.$ mixture, air $)$. Operated at $900 \mathrm{~V}$.

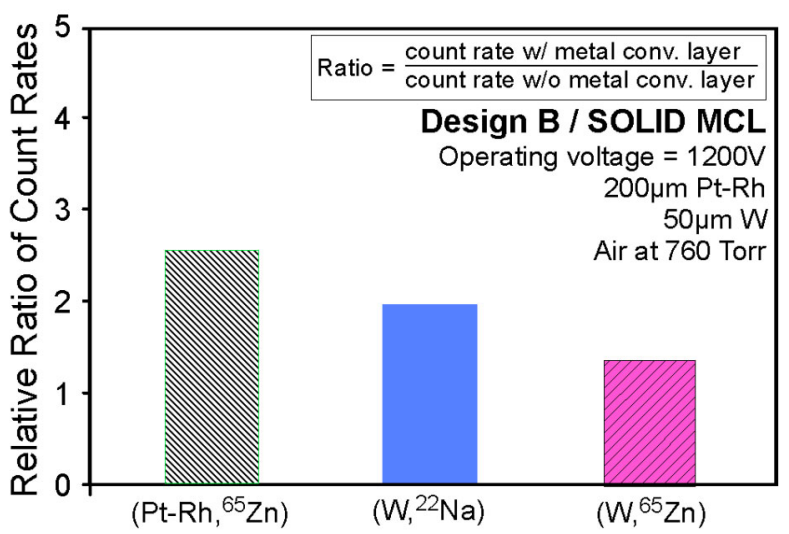

Fig. 8: Comparison of the conversion efficiency for different

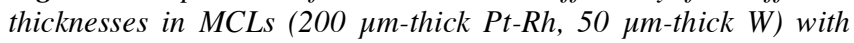
Design B. Applied voltage is $1200 \mathrm{~V}$ in ambient air. 


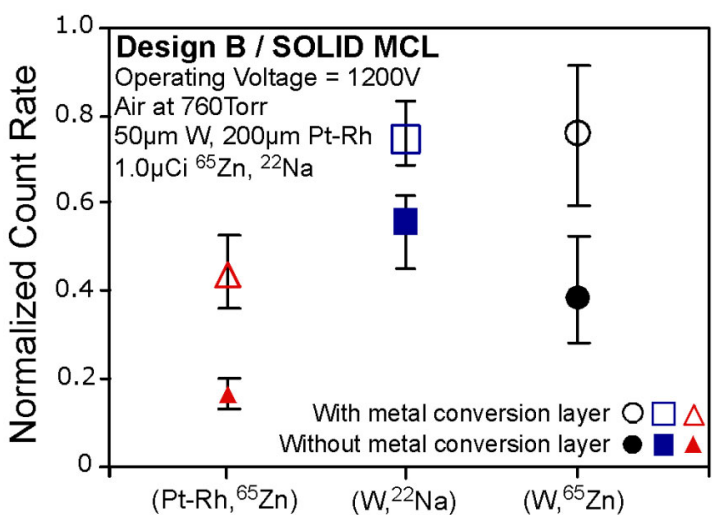

Fig. 9: Comparing normalized count rates from Design $B$ with unpatterned MCLs of Pt-Rh and W, in the presence of different isotopes. Error bars indicate the range of measured count rates.

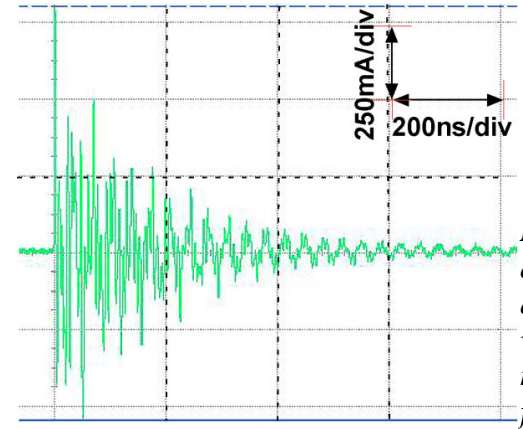

(a)

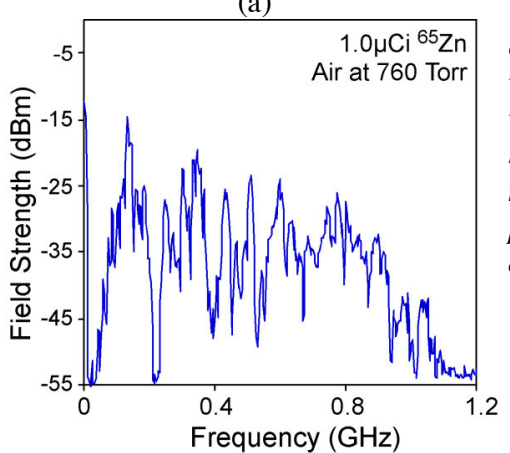

(b)

Design $B$ was tested in an air environment at 760 Torr. Higher conversion efficiencies (higher relative count ratios) were measured for $200 \mu \mathrm{m}$-thick Pt-Rh over $50 \mu \mathrm{m}$-thick $\mathrm{W}$, because interaction probability increases with layer thickness (Fig. 8). Higher count ratios for Design $B$ were measured while detecting ${ }^{22} \mathrm{Na}$ compared to ${ }^{65} \mathrm{Zn}$, because ${ }^{22} \mathrm{Na}$ emits (on average) $5 \mathrm{X}$ more gamma-rays per decay than ${ }^{65} \mathrm{Zn}$. Figure 9 compares the normalized count rates for Design $B$ and shows the range of statistical variation in count rates. A consistently higher count rate with an MCL shows the efficacy of the gamma-to-electron conversion method. For Design B, the overall count rates were lower with the Pt-Rh SOLID MCL compared to the W SOLID MCL. However, there was an overall improvement in operational stability with the Pt-Rh when compared to Design $B$ with W. In the absence of an MCL (using Design $B$ ), a higher relative count rate was observed in the proximity of ${ }^{22} \mathrm{Na} \quad(\approx 0.55)$ compared to ${ }^{65} \mathrm{Zn} \quad(\approx 0.38)$, because ${ }^{22} \mathrm{Na}$ has a higher rate of beta emission $(90 \%)$ compared to ${ }^{65} \mathrm{Zn}(1.4 \%)$. $A$ glass substrate was used for preliminary tests, but future efforts can use thin mica for increased photoelectron detection efficiency.
Current pulses (of duration $\approx 600 \mathrm{~ns}$ ) were measured with a wideband oscilloscope and the wireless signal emitted during sensor operation in the proximity of ${ }^{65} \mathrm{Zn}$ was received remotely using a spectrum analyzer and antenna positioned $\approx 30 \mathrm{~cm}$ from the detector (Fig. 10). The measured bandwidth extended beyond 1.2 GHz. The MCL showed minimal impact on the resulting RF field strength.

\section{CONCLUSIONS}

The proposed concept for a wireless micromachined gamma radiation sensor has been experimentally validated. The gas-based detector includes a layer of bulk metal on top of a glass substrate containing metal electrodes. The bulk metal converts gamma radiation to photoelectrons that ionize the gas medium, resulting in a current pulse. These fast current pulses generate a wideband wireless signal $(>1.2 \mathrm{GHz})$. The impact of two different structures patterned in the MCL was tested: TRENCH MCL and COLUMN MCL. The TRENCH MCL showed a count rate exceeding 28 $\mathrm{kcpm}$ and a $>10 \mathrm{X}$ improvement in sensing when compared to an un-patterned MCL. The detector operated under partial pressure in an $\mathrm{N}_{2} / \mathrm{Ne}$ mixture in the proximity of $1.0 \mu \mathrm{Ci}$ of ${ }^{22} \mathrm{Na}$. Operation in air with Pt-Rh and W MCLs located on the backside of the glass substrate showed a higher count rate with the MCL than without.

Overall, this work has shown that integrating micromachined metal layers for enhanced gamma detection efficiency provides important new functionality to miniaturized wireless gas-based sensors for beta particles. In doing so, it enables longer detection distances and detection of concealed nuclear materials. In the future, the metal conversion layer can be integrated into the gas packaging for the sensor.

\section{ACKNOWLEDGEMENTS}

This work was supported primarily by the Engineering Research Centers Program of the National Science Foundation under Award Number EEC-9986866. The facilities used for this research include the Michigan Nanofabrication Facility (MNF) at the University of Michigan. Y.G. acknowledges support through the IR/D program while working at the National Science Foundation. The findings do not necessarily reflect the views of the NSF. The authors would like to thank Mark T. Richardson for his help in machining the metal conversion layers.

\section{REFERENCES}

[1] C.K. Eun et al, "A magnetically enhanced 3-electrode wireless micro-Geiger counter," IEEE MEMS, Jan. 2007.

[2] B. Shafrir and A. Seidman, "High efficiency gamma-ray metal converters," Nucl. Instr. \& Meth., vol. 129, 1975 pp. 177-186.

[3] U. Shimoni et al., "Investigations on metal converters for gammaray detection and mapping," Nucl. Instr. \& Meth., vol. 117, 1974, pp. $599-603$.

[4] M. Nakamura, "The energy spectra and the quantum efficiencies of electrons emitted from the metallic elements irradiated by $60 \mathrm{Co}$ gamma-rays," J. Appl. Phys., 54(6) Jun. 1983, pp. 3141-3149.

[5] J.O. Hirschfelder et al., "The penetration of gamma-radiation through thick layers," Phys. Rev., 73(8), Apr. 1948, pp. 852-862.

[6] J. Martin, Physics of Radiation Protection, 2nd Ed., Ch.3, Wiley$\mathrm{VCH}, 2006$, Appendix B\&D.

[7] M.J. Berger et. al, "XCOM: Photon Cross Sections Database," NIST Standard Reference Database, Mar. 1998. http://physics.nist.gov/PhysRefData/Xcom/Text/XCOM.html

[8] H.H. Anderso, et. al, "ESTAR: Stopping power and range tables for electrons," NIST Standard Reference Database, http://physics.nist.gov/PhysRefData/Star/Text/ESTAR.html

[9] M.T. Richardson and Y.B. Gianchandani, "Achieving precision in high density batch mode micro-electro-discharge machining," $J$. Micromech. Microeng., vol. 18, pp. 1-12, 2008. 